\title{
On the Existence of a Point Subset with 3 or 6 Interior Points
}

\author{
Banyat Sroysang \\ Department of Mathematics and Statistics, Faculty of Science and Technology, Thammasat University, \\ Pathumthani 12121, Thailand
}

Correspondence should be addressed to Banyat Sroysang; banyat@mathstat.sci.tu.ac.th

Received 11 September 2013; Accepted 31 October 2013

Academic Editors: S. Hernández, A. Stipsicz, and S. Troubetzkoy

Copyright @ 2013 Banyat Sroysang. This is an open access article distributed under the Creative Commons Attribution License, which permits unrestricted use, distribution, and reproduction in any medium, provided the original work is properly cited.

For any finite planar point set $P$ in general position, an interior point of the set $P$ is a point of the set $P$ such that it is not on the boundary of the convex hull of the set $P$. For any positive integer $k \geq 3$, let $h(k)$ be the smallest integer such that every finite planar point set $P$ with no three collinear points and with at least $h(k)$ interior points has a subset $Q$ for which the interior of the convex hull of the set $Q$ contains exactly $k$ or $k+3$ interior points of the set $P$. In this paper, we prove that $h(3)=8$.

\section{Introduction}

In this paper, we focus on finite planar point sets in general position; that is, no three points are collinear. In 1935, Erdős and Szekeres [1] posed a problem: for any integer $k \geq 3$, determine the smallest positive integer $f(k)$ such that any finite point set of at least $f(k)$ points has a subset of $k$ points whose convex hull contains exactly $k$ vertices. In 1961, they [2] showed that $f(k) \geq 2^{k-2}+1$ for all integer $k \geq 3$ and then conjectured that $f(k) \geq 2^{k-2}+1$ for all integer $k \geq 3$. In 1974, Bonnice [3] proved that $f(3)=3$ and $f(4)=5$. In 1970, Kalbfleisch et al. [4] showed that $f(5)=9$. In 2006, the computer solution for $k=6$ was presented by Szekeres and Peters [5]; that, $f(6)=17$.

In 2001, Avis et al. [6] posed an interior point problem: for any integer $k \geq 1$, determine the smallest positive integer $g(k)$ such that any finite point set $P$ of at least $g(k)$ points has a subset $Q$ for which the interior of the convex hull of the set $Q$ contains exactly $k$ points in the set $P$. Moreover, they also showed the results that $g(1)=1$ and $g(2)=4$. In 1974, Bonnice [3] showed that $g(k) \geq 3 k-1$ for all integer $k \geq 3$. In 2008, Wei and Ding [7] showed that $g(k) \geq 3 k$ for all integer $k \geq 3$. Moreover, in 2009, they [8] also showed that $g(3)=9$. In 2011, Sroysang [9] showed that $g(k) \geq 4 k$ for all integer $k \geq 4$. Moreover, in 2012, he [10] also showed that $g(k) \geq k^{2}$ for all integer $k \geq 4$.
In 2001, Avis et al. [6] proved that 3 is the smallest positive integer such that any finite point set $P$ of at least 3 interior points has a subset $Q$ for which the interior of the convex hull of the set $Q$ contains exactly 3 or 4 points in the set $P$. Moreover, they [11] also proved that 7 is the smallest positive integer such that any finite point set $P$ of at least 7 interior points has a subset $Q$ for which the interior of the convex hull of the set $Q$ contains exactly 4 or 5 points in the set $P$. In 2009, Wei and Ding [12] showed that any planar point set $P$ with 3 vertices and 9 interior points has a subset with 5 or 6 interior points of the set $P$.

In 2010, Wei et al. [13] proved that 8 is the smallest positive integer such that any finite point set $P$ of at least 8 interior points has a subset $Q$ for which the interior of the convex hull of the set $Q$ contains exactly 3 or 5 points in the set $P$.

In 2012, Sroysang [14] proved that 7 is the smallest positive integer such that any finite point set $P$ of at least 7 interior points has a subset $Q$ for which the interior of the convex hull of the set $Q$ contains exactly 3 or 7 points in the set $P$.

In this paper, we pose an interior point problem: for any integer $k \geq 3$, determine the smallest positive integer $h(k)$ such that any finite point set $P$ of at least $h(k)$ points has a subset $Q$ for which the interior of the convex hull of the set $Q$ contains exactly $k$ or $k+3$ points in the set $P$. We show that $h(3)=8$; that is, 8 is the smallest positive integer such that any finite point set $P$ of at least 8 interior points has a subset $Q$ for which the interior of the convex hull of the set $Q$ contains exactly 3 or 6 points in the set $P$. 


\section{Preliminaries}

In this section, we list propositions and notations about the set $P$, where $P$ is a finite planar point set such that no three points are collinear.

An interior point of the set $P$ is a point of the set $P$ such that it is not on the boundary of the convex hull of the set $P$.

We denote notations as follows:

$I(P):=$ the set of interior points of the set $P$,

$i(P):=$ the number of elements in the set $I(P)$,

$C H(P):=$ the convex hull of the set $P$,

$\operatorname{int} C H(P):=$ the interior of the set $C H(P)$,

$V(P):=$ the set of vertices of the set $C H(P)$,

$v(P):=$ the number of elements in the set $V(P)$.

For $Q \subseteq P$,

$I^{*}(Q):=I(P) \cap \operatorname{int} C H(Q)$,

$i^{*}(Q):=$ the number of elements in the set $I^{*}(Q)$.

For $x, y, z \in P$,

$\Delta x y z:=$ the triangle with vertices $x, y$, and $z$.

An edge of the set $P$ is an edge in $C H(P)$. A subset $Q$ of the set $P$ is called a $k$-int subset if $i^{*}(Q)=k$.

Note that there is $Q \subseteq P$ such that $i(Q) \neq i^{*}(Q)$.

Proposition 1 (see [8]). 9 is the smallest integer such that any finite point set $P$ of at least 9 interior points has a subset $Q$ for which the interior of the convex hull of the set $Q$ contains exactly 3 points in the set $P$.

For any positive integer $k \geq 3$, we let $h(k)$ be the smallest integer such that every planar point set $P$ with no three collinear points and with at least $h(k)$ interior points has a subset $Q$ for which the interior of the convex hull of the set $Q$ contains exactly $k$ or $k+3$ points of the set $P$.

For any positive integer $k \geq 3$,

$h(k)=\min \left\{s: i(P) \geq s \Longrightarrow \exists Q \subseteq P\right.$ s.t. $i^{*}(Q)=k$ or $\left.k+3\right\}$.

A finite planar point set $P$ is called a deficient point set of type $P(m, s, k, n)$ and denoted by $P=P(m, s, k, n)$ if $v(P)=$ $m, i(P)=s$, and $i^{*}(Q) \notin\{k, n\}$ for all $Q \subseteq P$.

An edge $x y$ of the set $P(3, s, 3,3)$ is of type $k$ if there exists a subset $Q$ of the set $P$ with $i^{*}(Q)=k$ such that the edge $x y$ is an edge of the set $Q$.

Proposition 2 (see [8]). Every edge of a deficient point set of type $P(3,7,3,3)$ is of type 2 .

\section{Main Results}

In this section, we will show that 8 is the smallest positive integer such that any finite point set $P$ of at least 8 interior points has a subset $Q$ for which the interior of the convex hull of the set $Q$ contains exactly 3 or 6 points in the set $P$.

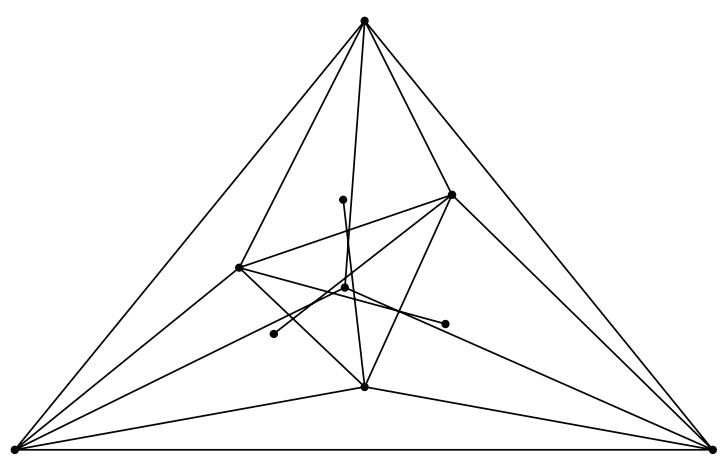

Figure 1: A deficient point set of type $P(3,7,3,6)$.

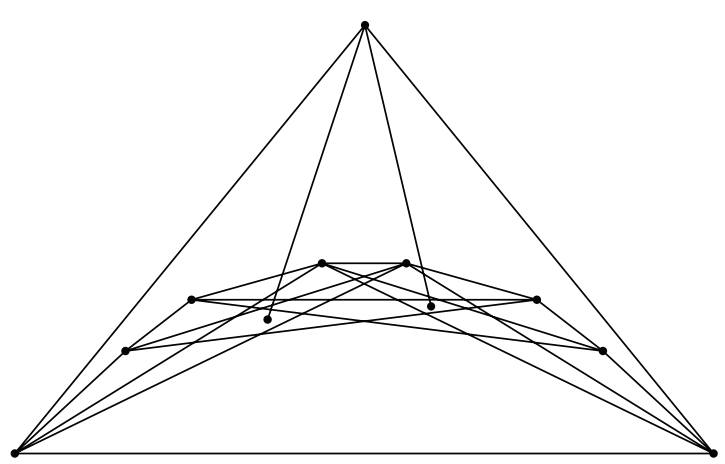

Figure 2: 8-I-monster.

Lemma 3. $h(3) \leq 9$.

Proof. Let $P$ be a finite planar point set such that $i(P) \geq 9$.

By Proposition 1, there is a subset $Q$ of the set $P$ such that $i^{*}(Q)=3$. Then $i^{*}(Q) \in\{3,6\}$. Hence, $h(3) \leq 9$.

Lemma 4. $h(3) \geq 8$.

Proof. This suffices to show the existence of a deficient point set of type $P(3,7,3,6)$. We construct a deficient point set $P$ of type $P(3,7,3,6)$ as shown in Figure 1 . Hence, $h(3) \geq 8$.

Lemma 5. Let $P$ be a finite planar point set. Assume that $v(P)=3$ and $i(P)=8$. Then the set $P$ has a 3-int or 6-int subset.

Proof. Suppose that each subset of a planar point set $P$ is not a 3-int subset. In [8], we have only three different configurations of the type $P(3,8,3,3)$ as shown in Figures 2, 3, and 4 . However, each configuration has a subset $Q$ for which the interior of the convex hull of the set $Q$ contains exactly 6 points of the set $P$. Hence, the set $P$ has a 3 -int or 6 -int subset.

Lemma 6. Let $P$ be a finite planar point set. Assume that $v(P)=4$ and $i(P)=8$. Then the set $P$ has a 3-int or 6-int subset.

Proof. Let $V(P)=\{x, y, z$, and $w\}$ be such that vertices $x, y$, $z, w$ are put into counterclockwise positions, respectively (see Figure 5). 


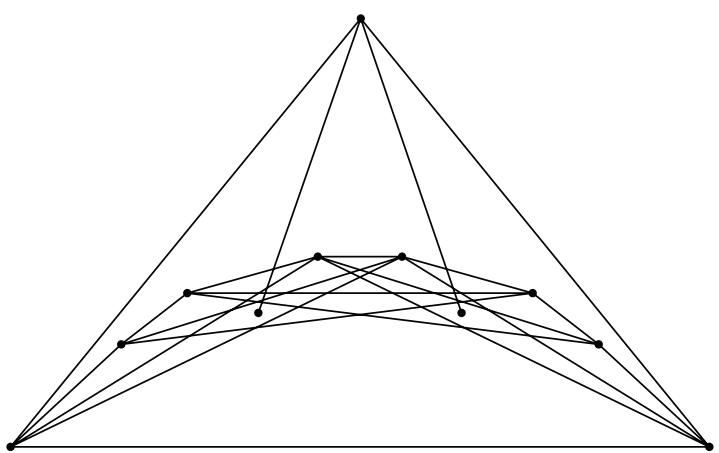

Figure 3: 8-II-monster.

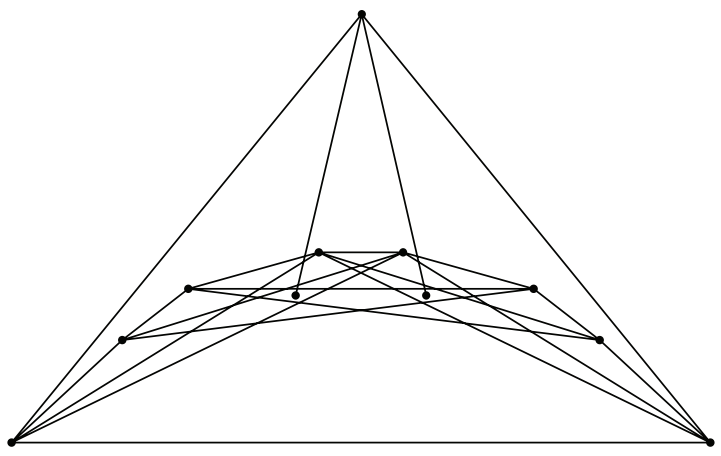

FIgURE 4: 8-III-monster.

Suppose that each subset of the planar point set $P$ is not a 6-int subset. Then the sets $\Delta x y z, \Delta y z w, \Delta z w x$, and $\Delta w x y$ are not 6-int subsets.

If $\Delta x y z$ is a 2 -int subset, then the set $\Delta z w x$ is a 6 -int subset. Then the set $\Delta x y z$ is not a 2-int subset. Similarly, the sets $\Delta y z w, \Delta z w x$, and $\Delta w x y$ are not 2-int subsets.

Let $T=\{\Delta x y z, \Delta y z w, \Delta z w x, \Delta w x y\}$.

To show that the set $P$ has a 3 -int subset, we divide into seven cases.

Case 1. There is an element $A$ in the set $T$ such that the set $A$ is a 3 -int subset.

In this case, the set $P$ has a 3 -int subset.

Case 2. There is an element $A$ in the set $T$ such that the set $A$ is a 5 -int subset.

Without loss of generality, we assume that $A=\Delta x y z$. Then the set $\Delta z w x$ is a 3 -int subset. Thus, the set $P$ has a 3-int subset.

Case 3. There is an element $A$ in the set $T$ such that the set $A$ is a 7 -int subset.

Without loss of generality, we assume that $A=\Delta x y z$. Then the set $\Delta z w x$ is a 1-int subset. If the set $A$ has a 3-int subset, then the set $P$ has a 3 -int subset. Assume that the set $A$ is a deficient point set of type $P(3,7,3,3)$. By Proposition 2 , there is a subset $B$ of the set $C H(\Delta x y z)$ with $i^{*}(B)=2$ such

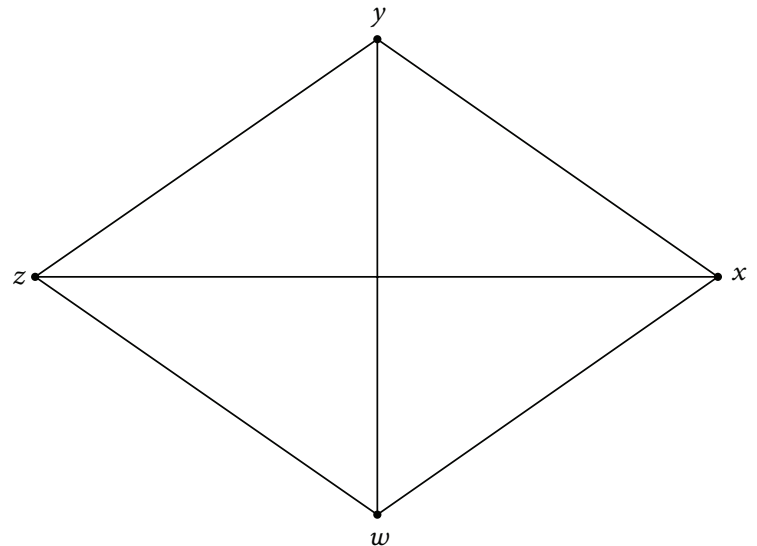

FIGURE 5: Vertices $x, y$, and $z, w$ are put into counterclockwise positions.

that the edge $x z$ is an edge of the set $B$. Let $Q=B \cup \Delta z w x$. Then $i^{*}(Q)=3$. Thus, the set $P$ has a 3 -int subset.

Case 4. There is an element $A$ in the set $T$ such that the set $A$ is a 1-int subset.

Without loss of generality, we assume that $A=\Delta x y z$. Then the set $\Delta z w x$ is a 7-int subset. Similar to Case 3, the set $P$ has a 3-int subset.

Case 5. There is an element $A$ in the set $T$ such that the set $A$ is an 8-int subset.

By Lemma 5, the set $P$ has a 3 -int subset.

Case 6. There is an element $A$ in the set $T$ such that the set $A$ is a 0 -int subset.

Without loss of generality, we assume that $A=\Delta x y z$. Then the set $\Delta z w x$ is an 8 -int subset. By Lemma 5 , the set $P$ has a 3-int subset.

Case 7. The sets $\Delta x y z, \Delta y z w, \Delta z w x$, and $\Delta w x y$ are 4-int subsets.

If one of them has a 3-int subset, then the set $P$ has a 3-int subset. Assume that they are deficient point sets without a 3int subset. If the edge $x z$ of the set $\Delta x y z$ is of type 2 , then we obtain that $i^{*}(P \backslash\{y\})=6$. It follows that, the edge $x z$ of the set $\Delta x y z$ is of type 0 or type 1 . If the edge $x z$ of the set $\Delta x y z$ is of type 0 , then the edge $x y$ of the set $\Delta x y z$ is of type 3, so the set $P$ has a 3 -int subset. Next, we will assume that the edge $x z$ of the set $\Delta x y z$ is of type 1. Similarly, it suffices to assume that the edge $x z$ of the set $\Delta z w x$ is only of type 1, the edge $y w$ of the set $\Delta y z w$ is only of type 1, and the edge $y w$ of the set $\Delta w x y$ is only of type 1 . Hence, we obtain only one possible configuration as shown in Figure 6.

However, there is a subset $Q$ of $P$ such that $i^{*}(Q)=3$, as shown in Figure 7 . Thus, the set $P$ has a 3 -int subset.

Therefore, the set $P$ has a 3 -int or 6 -int subset. This proof is completed.

Lemma 7. Let $P$ be a finite planar point set. Assume that $v(P) \geq 5$ and $i(P)=8$. Then the set $P$ has a 3-int or 6-int subset. 


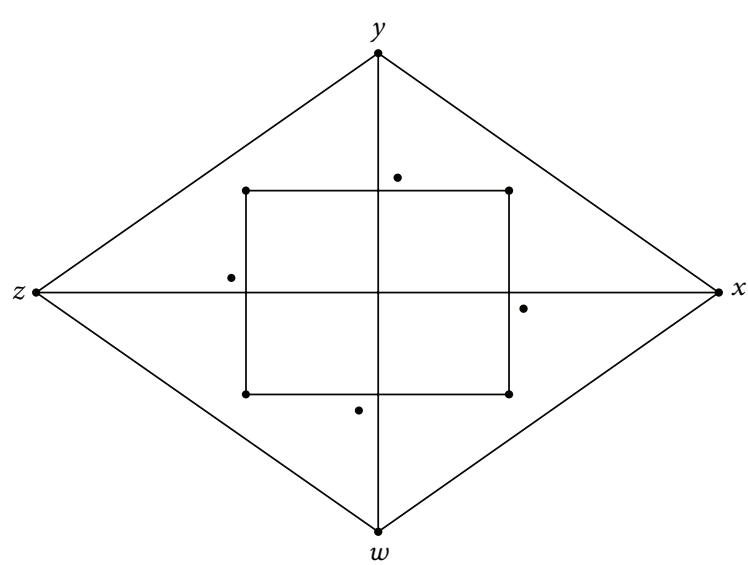

Figure 6: The edges $x z$ and $y w$ are only of type 1 .

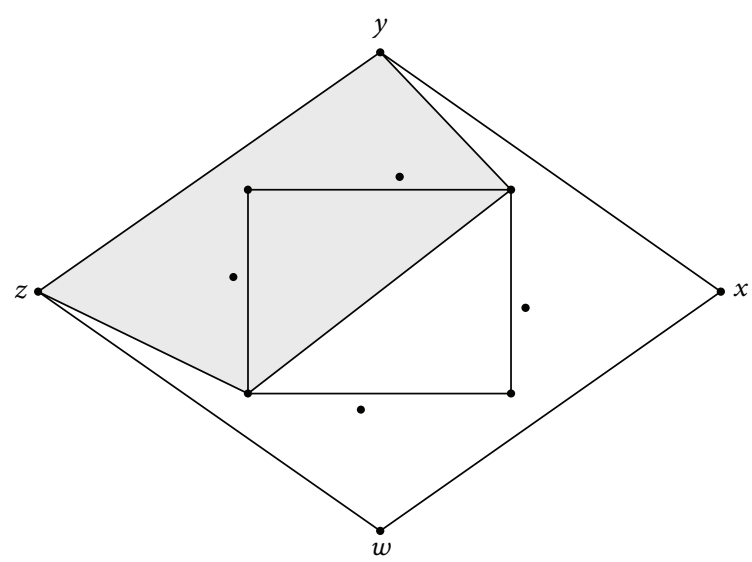

Figure 7: A 3-int subset of the set in Figure 6.

Proof. Let $v(P)=m$ and $V(P)=\left\{v_{1}, v_{2}, \ldots, v_{m}\right\}$ be such that vertices $v_{1}, v_{2}, \ldots, v_{m}$ are put into counterclockwise positions, respectively (see in Figure 8 ).

Suppose that each subset of the set $P$ is not a 6-int subset. Then the set $\Delta v_{1} v_{j} v_{j+1}$ is not a 6-int subset for all $j$.

Let $T=\left\{\Delta v_{1} v_{j} v_{j+1} \mid j=2,3, \ldots, m\right\}$.

To show that the set $P$ has a 3 -int subset, we divide into six cases.

Case 1. There is an element $A$ in the set $T$ such that the set $A$ is a 3 -int subset.

In this case, the set $P$ has a 3 -int subset.

Case 2. There is an element $A$ in the set $T$ such that the set $A$ is a 7 -int subset.

Then the set $\Delta v_{1} v_{t} v_{t+1}$ is a 1-int subset for some $t$. Without loss of generality, we assume $A=\Delta v_{1} v_{j} v_{j+1}$ for some $j>t$. If the set $A$ has a 3 -int subset, then the set $P$ has a 3-int subset. Assume that the set $A$ is a deficient point set of type $P(3,7,3,3)$. By Proposition 2 , there is a subset $B$ of the set $C H(A)$ with $i^{*}(B)=2$ such that the edge $v_{1} v_{j}$ is an edge of the set $B$. Let $Q=B \cup \Delta v_{1} v_{t} v_{t+1}$. Then $i^{*}(Q)=3$. Thus, the set $P$ has a 3 -int subset.

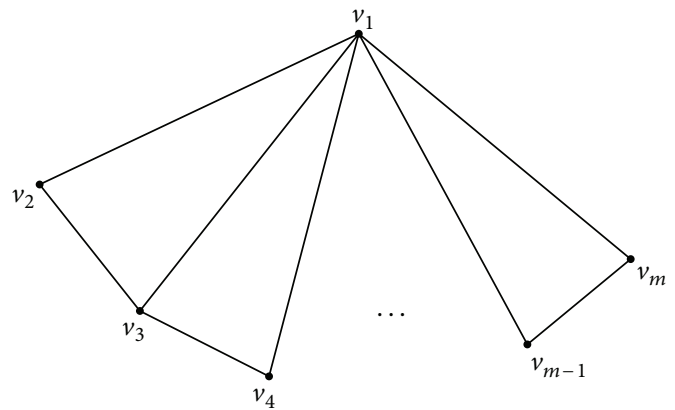

FIgURE 8: Vertices $v_{1}, v_{2}, \ldots, v_{m}$ are put into counterclockwise positions.

Case 3. There is an element $A$ in the set $T$ such that the set $A$ is a 5 -int subset.

We divide into three subcases.

Subcase 3.1. There is an element $B$ in the set $T$ such that the set $B$ is a 3 -int subset.

In this subcase, the set $P$ has a 3 -int subset.

Subcase 3.2. There exist elements $B, C$, and $D$ in the set $T$ such that the sets $B, C$, and $D$ are 1-int subsets.

It follows that $A=\Delta v_{1} v_{j} v_{j+1}, B=\Delta v_{1} v_{t} v_{t+1}, C=$ $\Delta v_{1} v_{s} v_{s+1}$, and $D=\Delta v_{1} v_{r} v_{r+1}$, where $j, t, s, r \in\{2,3, \ldots, m\}$. Without loss of generality, we assume that $|t-j|=\min \{|t-j|$, $|s-j|,|r-j|\}$. Then the set $A \cup B$ is a 6 -int subset. This is impossible.

Subcase 3.3. There exist elements $B, C$ in the set $T$ such that the set $B$ is a 1-int subset and the set $C$ is a 2-int subset.

It follows that $A=\Delta v_{1} v_{j} v_{j+1}, B=\Delta v_{1} v_{t} v_{t+1}$ and $C=$ $\Delta v_{1} v_{r} v_{r+1}$ where $j, t, r \in\{2,3, \ldots, m\}$. If $r$ is not between $t$ and $j$, then the set $A \cup B$ is a 6-int subset which is impossible. Thus, $r$ is between $t$ and $j$. We choose $Q=B \cup C$. Then $i^{*}(Q)=3$. Hence, the set $P$ has a 3 -int subset.

Case 4. There is an element $A$ in the set $T$ such that the set $A$ is a 4 -int subset.

We divide into five subcases.

Subcase 4.1. There is an element $B$ in the set $T$ such that the set $B$ is a 3 -int subset.

In this subcase, the set $P$ has a 3 -int subset.

Subcase 4.2. There exist elements $B, C, D$, and $E$ in the set $T$ such that the sets $B, C, D$, and $E$ are 1-int subsets.

It follows that $A=\Delta v_{1} v_{j} v_{j+1}, B=\Delta v_{1} v_{t} v_{t+1}, C=$ $\Delta v_{1} v_{s} v_{s+1}, D=\Delta v_{1} v_{k} v_{k+1}$, and $E=\Delta v_{1} v_{r} v_{r+1}$, where $j, t, s$, $r, k \in\{2,3, \ldots, m\}$. Without loss of generality, we can assume that $t<s<k<r$. If $j<s$, then the set $C \cup D \cup E$ is a 3-int subset. If $k<j$, then the set $B \cup C \cup D$ is a 3 -int subset. Thus, the set $P$ has a 3 -int subset if $j<s$ or $k<j$. Next, we will show that the statement " $s<j<k$ " is impossible. We suppose that $s<j<k$. Then the set $A \cup C \cup D$ is a 6-int subset which is a contradiction. 
Subcase 4.3. There exist elements $B$ and $C$ in the set $T$ such that the sets $B$ and $C$ are 2 -int subsets.

It follows that $A=\Delta v_{1} v_{j} v_{j+1}, B=\Delta v_{1} v_{t} v_{t+1}$ and $C=$ $\Delta v_{1} v_{r} v_{r+1}$ where $j, t, r \in\{2,3, \ldots, m\}$. Without loss of generality, we assume $|t-j|<|r-j|$. Then the set $A \cup B$ is a 6-int subset. This is impossible.

Subcase 4.4. There exist elements $B, C$, and $D$ in the set $T$ such that the sets $B$ and $C$ are 1-int subsets and the set $D$ is a 2-int subset.

It follows that $A=\Delta v_{1} v_{j} v_{j+1}, B=\Delta v_{1} v_{t} v_{t+1}, C=$ $\Delta v_{1} v_{s} v_{s+1}$, and $D=\Delta v_{1} v_{r} v_{r+1}$, where $j, t, s, r \in\{2,3, \ldots, m\}$. Without loss of generality, we can assume that $j<r$. Let $k=\max \{t, s\}$ and $l=\min \{t, s\}$. If $k<r$, then the set $A \cup B \cup C$ is a 6-int subset. If $r<l$, then the set $A \cup D$ is a 6-int subset. Thus, we obtain that $l<r<k$. Then the set $D \cup \Delta v_{1} v_{k} v_{k+1}$ is a 3 -int subset. Hence, the set $P$ has a 3 -int subset.

Subcase 4.5. There is an element $B$ in the set $T \backslash\{A\}$ such that the set $B$ is a 4 -int subset.

Without loss of generality, we assume that $A=\Delta v_{1} v_{j} v_{j+1}$ and $B=\Delta v_{1} v_{t} v_{t+1}$, where $j<t$. Let $C=\left\{v_{1}, v_{j}, v_{t}, v_{t+1}\right\}$ and $D=\left\{v_{1}, v_{j}, v_{j+1}, v_{t+1}\right\}$. Then the sets $C$ and $D$ are not 6-int subsets. If the set $C$ is an 8 -int subset or the set $B$ is an 8-int subset then, by Lemma 6 , the set $P$ has a 3 -int subset. If the set $C$ is a 7-int subset, then the set $\Delta v_{1} v_{j} v_{t}$ is a 3-int subset, so the set $P$ has a 3 -int subset. If the set $D$ is a 7-int subset, then the set $\Delta v_{1} v_{j+1} v_{t+1}$ is a 3 -int subset, so the set $P$ has a 3 -int subset. If the set $C$ is a 5 -int subset, then the set $\Delta v_{j} v_{j+1} v_{t}$ is a 3-int subset, so the set $P$ has a 3-int subset. If the set $D$ is a 5-int subset, then the set $\Delta v_{j+1} v_{t} v_{t+1}$ is a 3-int subset, so the set $P$ has a 3 -int subset. Next, we assume that the sets $C$ and $D$ are 4 -int subsets. Then $\Delta v_{1} v_{j} v_{t+1}$ is a 0 -int subset. Then the set $\left\{v_{j}, v_{j+1}, v_{t}, v_{t+1}\right\}$ is an 8 -int subset. By Lemma 6, the set $P$ has a 3 -int subset.

Case 5. There is an element $A$ in the set $T$ such that the set $A$ is an 8 -int subset.

By Lemma 5, the set $P$ has a 3 -int subset.

Case 6. We have $i^{*}(A) \leq 2$ for all $A \in T$.

If there exist elements $A, B, C$, and $D$ in the set $T$ such that the sets $A, B, C$, and $D$ are 2-int subsets where the sets $A, B, C$, and $D$ put into anticlockwise positions, then the set $A \cup B \cup C$ is a 6-int subset. Thus, we obtain that there is an element in the set $T$ such that it is a 1-int subset. It is easy to see that $P$ has a 3 -int subset.

Therefore, the set $P$ has a 3 -int or 6-int subset. This proof is completed.

Theorem 8. One has $h(3)=8$.

Proof. By Lemmas 3 and 4 , it follows that $8 \leq h(3) \leq 9$. By Lemmas 5, 6, and 7, we obtain that $h(3) \leq 8$. Hence, $h(3)=8$.

\section{Conclusion and Discussion}

In [6], 3 is the smallest positive integer such that any finite point set $P$ of at least 3 interior points has a subset $Q$ for which the interior of the convex hull of the set $Q$ contains exactly 3 or 4 points in the set $P$.

In [13], 7 is the smallest positive integer such that any finite point set $P$ of at least 7 interior points has a subset $Q$ for which the interior of the convex hull of the set $Q$ contains exactly 3 or 5 points in the set $P$.

In this paper, 8 is the smallest positive integer such that any finite point set $P$ of at least 8 interior points has a subset $Q$ for which the interior of the convex hull of the set $Q$ contains exactly 3 or 6 points in the set $P$.

In [14], 7 is the smallest positive integer such that any finite point set $P$ of at least 7 interior points has a subset $Q$ for which the interior of the convex hull of the set $Q$ contains exactly 3 or 7 points in the set $P$.

In [15], 8 is the smallest positive integer such that any finite point set $P$ of at least 8 interior points has a subset $Q$ for which the interior of the convex hull of the set $Q$ contains exactly 3 or 8 points in the set $P$. Moreover, 9 is the smallest positive integer such that any finite point set $P$ of at least 9 interior points has a subset $Q$ for which the interior of the convex hull of the set $Q$ contains exactly 3 or $k$ points in the set $P$, where $k \geq 9$.

For any positive integer $k \geq 4$, we let $h^{*}(k)$ be the smallest integer such that every planar point set $P$ with no three collinear points and with at least $h^{*}(k)$ interior points has a subset $Q$ for which the interior of the convex hull of the set $Q$ contains exactly 3 or $k$ points of the set $P$.

For any positive integer $k \geq 3$,

$$
h^{*}(k)=\min \left\{s: i(P) \geq s \Longrightarrow \exists Q \subseteq P \text { s.t. } i^{*}(Q)=3 \text { or } k\right\} \text {. }
$$

Thus, we have the following formulas:

$$
\begin{array}{cc}
h^{*}(k)=3 & \text { if } k=4 ; \\
h^{*}(k)=7 & \text { if } k=5,7 ; \\
h^{*}(k)=8 & \text { if } k=6,8 ; \\
h^{*}(k)=9 & \text { if } k \geq 9 .
\end{array}
$$

\section{Conflict of Interests}

The author declares that there is no conflict of interests regarding the publication of this paper.

\section{Acknowledgment}

The author would like to thank the referees for their useful comments and suggestions.

\section{References}

[1] P. Erdős and G. Szekeres, "A combinatorial problem in geometry," Compositio Mathematica, vol. 2, pp. 463-470, 1935.

[2] P. Erdős and G. Szekeres, "On some extremum problems in elementary geometry," Annales Universitatis Scientiarum Budapestinensis de Rolando Eötvös Nominatae, vol. 3-4, pp. 53$62,1961$. 
[3] W. E. Bonnice, "On convex polygons determined by a finite planar set," The American Mathematical Monthly, vol. 81, pp. 749-752, 1974.

[4] J. D. Kalbfleisch, J. G. Kalbfleisch, and R. G. Stanton, "A combinatorial problem on convex $n$-gons," in Proceedings of the Louisiana Conference on Combinatorics, Graph Theory, and Computing, pp. 180-188, 1970.

[5] G. Szekeres and L. Peters, "Computer solution to the 17-point Erdős-Szekeres problem," The ANZIAM Journal, vol. 48, no. 2, pp. 151-164, 2006.

[6] D. Avis, K. Hosono, and M. Urabe, "On the existence of a point subset with a specified number of interior points," Discrete Mathematics, vol. 241, no. 1-3, pp. 33-40, 2001.

[7] X. Wei and R. Ding, "More on planar point subsets with a specified number of interior points," Mathematical Notes, vol. 83, no. 5-6, pp. 684-687, 2008.

[8] X. Wei and R. Ding, "More on an Erdős-Szekeres-type problem for interior points," Discrete \& Computational Geometry, vol. 42, no. 4, pp. 640-653, 2009.

[9] B. Sroysang, "A lower bound for Erdős-Szekeres-type problem with interior points," International Journal of Open Problems in Computer Science and Mathematics, vol. 4, no. 4, pp. 68-73, 2011.

[10] B. Sroysang, "An improved lower bound for an Erdős-Szekerestype problem with interior points," Applied Mathematical Sciences, vol. 6, no. 69-72, pp. 3453-3459, 2012.

[11] D. Avis, K. Hosono, and M. Urabe, "On the existence of a point subset with 4 or 5 interior points," in Discrete and Computational Geometry, vol. 1763 of Lecture Notes in Computer Science, pp. 57-64, Springer, Berlin, Germany, 2000.

[12] X. Wei and R. Ding, "A note on point subset with 5 or 6 interior points," Southeast Asian Bulletin of Mathematics, vol. 33, no. 6, pp. 1207-1214, 2009.

[13] X. Wei, W. Lan, and R. Ding, "On the existence of a point subset with three or five interior points," Mathematical Notes, vol. 88, no. 1-2, pp. 103-111, 2010.

[14] B. Sroysang, "On the existence of a point subset with 3 or 7 interior points," Applied Mathematical Sciences, vol. 6, no. 129132, pp. 6593-6600, 2012.

[15] B. Sroysang, "Remarks on point subset with 3 or $3+n$ interior points," Advances and Applications in Mathematical Sciences, vol. 10, no. 6, pp. 627-630, 2011. 


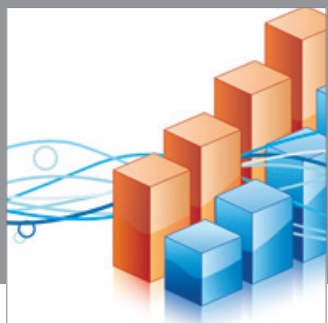

Advances in

Operations Research

mansans

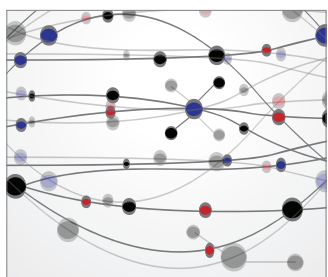

The Scientific World Journal
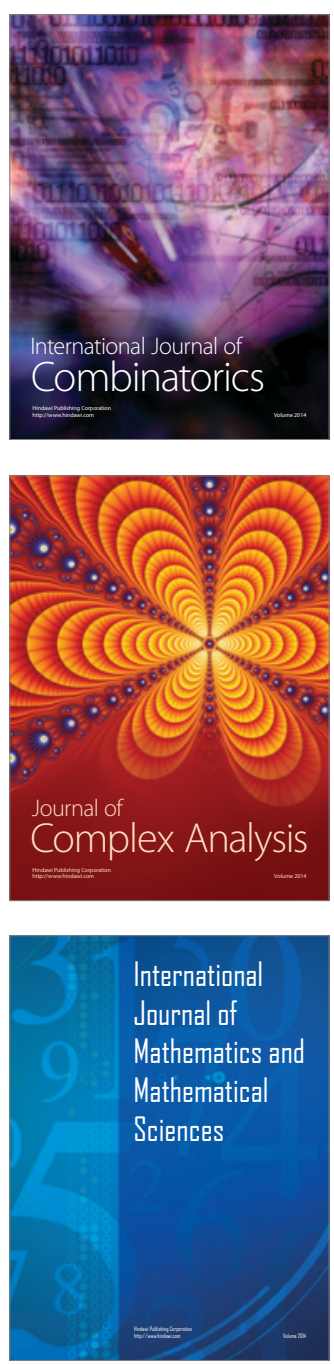
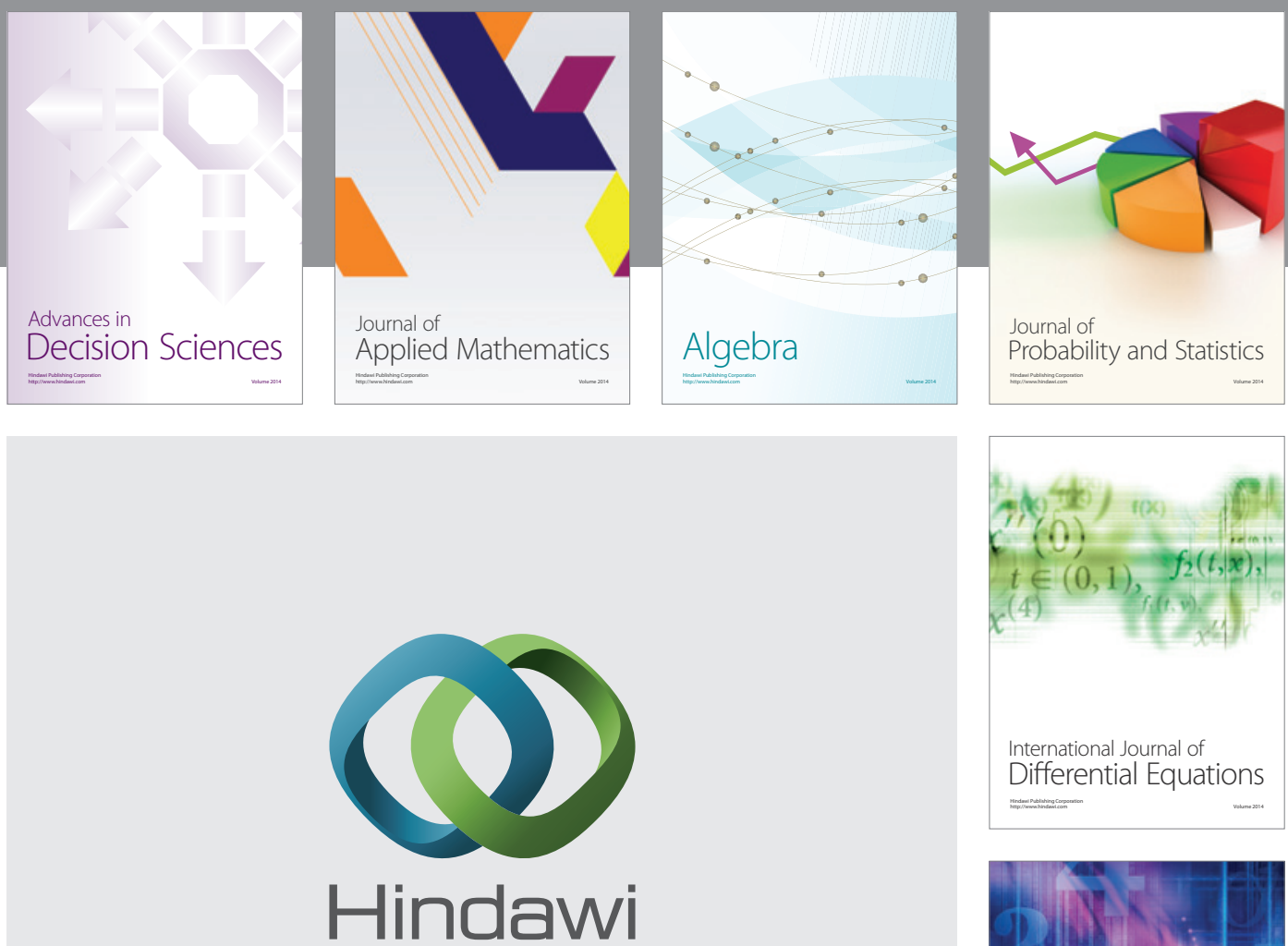

Submit your manuscripts at http://www.hindawi.com
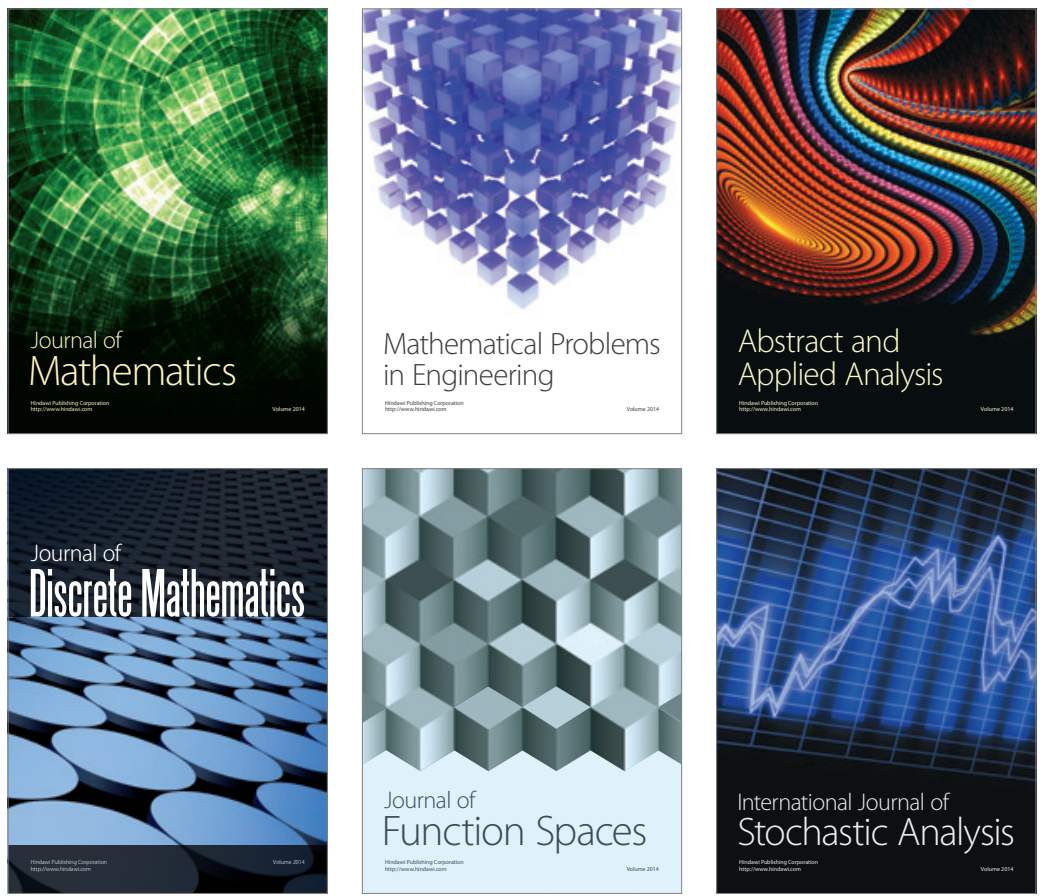

Journal of

Function Spaces

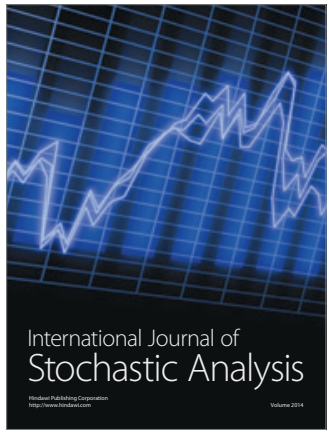

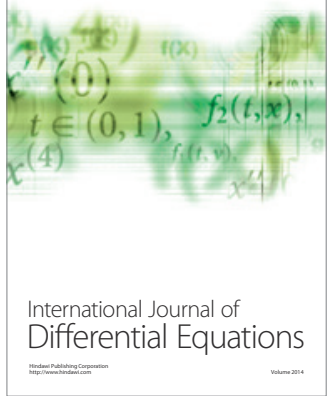
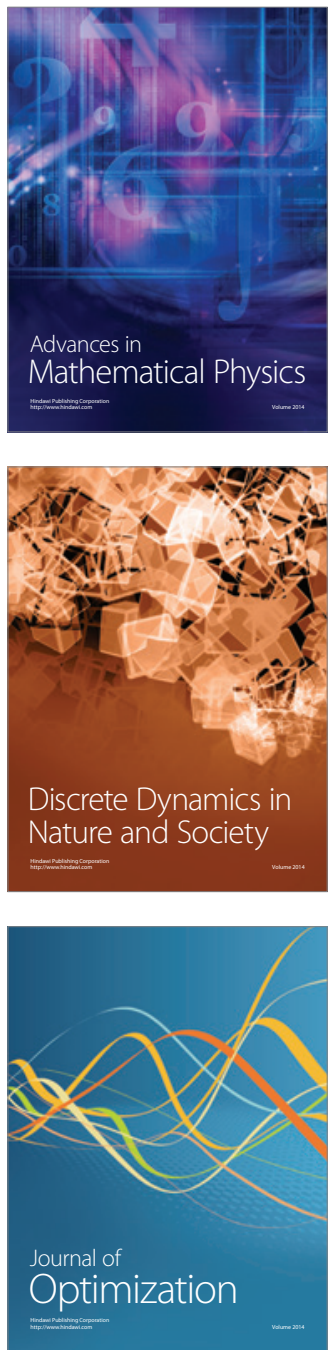